\title{
The validity of simple methods to detect poor nutritional status in paediatric oncology patients
}

\author{
Alexia J. Murphy ${ }^{1 *}$, Melinda White ${ }^{2}$ and Peter S.W. Davies ${ }^{1}$ \\ ${ }^{1}$ Discipline of Paediatrics and Child Health, Children's Nutrition Research Centre, Royal Children's Hospital, University of \\ Queensland, Herston 4029, Brisbane, QLD, Australia \\ ${ }^{2}$ Department of Nutrition and Dietetics, Royal Children's Hospital, Brisbane, QLD, Australia
}

(Received 3 January 2008 - Revised 8 August 2008 - Accepted 26 August 2008 - First published online 8 October 2008)

Poor nutritional status is a major concern for children being treated for an oncology condition. It is vital for optimal prognosis that nutritional concerns are recognised and treated promptly. The majority of oncology treatment centres only use simple methods to screen for poor nutritional status; however, it is unknown whether these simple methods accurately recognise poor nutritional status. We aimed to determine whether commonly used simple nutritional assessment variables could accurately identify poor nutritional status in children being treated for oncological conditions. This cross-sectional study measured height, weight, mid-upper arm circumference, triceps skinfold, albumin levels and body cell mass in forty children being treated for an oncological condition. To determine whether commonly used nutritional variables were suitable determinants, the variables were compared against the reference measure of body cell mass index (BCMI) Z-scores. Using the BCMI Z-score cut-off of $-1 \cdot 65$, $48 \%$ of the study population were considered poorly nourished. Correlational analysis showed that there was no significant biological relationship between the BCMI Z-score and the simple parameters. When divided into two groups based on the nutritional status indicated by the BCMI $Z$-score, the independent $t$ tests between the well-nourished and malnourished groups demonstrated that there was a significant difference in the BMI $Z$-score $(P=0 \cdot 01)$ between the groups. No simple nutritional measures were found to accurately identify poor nutritional status in children being treated for oncological conditions.

Poor nutritional status: Oncology: Body composition: Children

The prevalence of poor nutritional status at diagnosis and during therapy in children with an oncological condition has been reported to be as high as $60 \%$ depending on diagnosis, treatment and diagnostic techniques ${ }^{(1,2)}$. Previous studies have found that children who are either under- or overweight have a poorer prognosis compared with those who are well nourished $^{(3-7)}$. Poor nutritional status in children who are either under- or overweight, is associated with increased infection rate, decreased tolerance of chemotherapy, higher relapse rate and increased mortality in paediatric cancer patients ${ }^{(4-6)}$. Despite the concerns with poor nutritional status in the paediatric oncology population and its link to poor prognosis, not all centres complete nutritional assessment routinely ${ }^{(8)}$. Given the high prevalence of poor nutritional status and potential impact nutritional status can have on a child undergoing treatment for cancer, it is imperative that oncology centres regularly assess the nutritional status of their paediatric patients to ensure early identification and intervention.

Body cell mass (BCM) measurements by total body potassium (TBK) counting are an ideal indicator of poor nutritional status in oncology patients. The BCM is the metabolically active component of the fat-free mass and is an ideal indicator of nutritional status as it is the part of the body, which is vital for function, with poor nutritional status being defined as a loss of functional cell mass. BCM measurements are a good reflection of nutritional status in children with oncological conditions as they are independent of hydration changes that occur with disease ${ }^{(9)}$. Other body composition methods that rely on the assumption of a constant hydration will over- or underestimate body composition in this population.

Unfortunately, TBK measurements are not available in most clinical settings and are time-consuming methods, so the proposal of this measurement in the majority of oncology settings is impractical. To ensure that oncology centres will assess the nutritional status of patients, the methods need to be simple, inexpensive and be able to be performed by support staff. Some methods that fit these criteria, which are currently recommended in oncology settings, include measurements of weight, height, albumin, triceps skinfold and mid-upper arm circumference (MUAC) ${ }^{(8,10)}$. The calculation of ideal body weight and weight loss over 1 month has also been suggested as useful for assessment in this population ${ }^{(11)}$.

The problem with these simple measures is that the level of nutritional status, which is detected, has been shown to vary with the method used ${ }^{(12-14)}$. It is not known which simple measures, if any, accurately detect poor nutritional status in

Abbreviations: BCM, body cell mass; BCMI, body cell mass index; IBW, ideal body weight for height; MUAC, mid-upper arm circumference; TBK, total body potassium. *Corresponding author: Alexia J. Murphy, fax + 61733464684 , email alexia.murphy@uq.edu.au 
children being treated for oncological conditions. If the validity of these measures for assessing poor nutritional status cannot be proved, then oncology centres should question the application of simple measures for clinical management. Therefore, the aim of the present paper is to evaluate whether simple, commonly used nutritional assessment methods can accurately identify poor nutritional status, as determined by the BCM measurements, in children being treated by an oncology department.

\section{Material and methods}

\section{Subjects}

Subjects were children between 5 and 16 years, who were being treated for a range of oncological conditions or children with other syndromes requiring bone marrow transplant. All children were patients of the Oncology Department at the Royal Children's Hospital, Brisbane. The study protocol was approved by the University of Queensland Medical Research Committee and the Royal Children's Hospital Ethics Committee. Written consent was obtained from all parents and children over 12 years, while verbal assent was obtained for children under 12 years.

\section{Measurements}

All measurements were taken on the same day in the Body Composition Laboratory at the Royal Children's Hospital. Body weight was measured to the nearest $0.05 \mathrm{~kg}$ using calibrated digital scales (Tanita BWB-600; Wedderburn Scales, Brisbane, QLD, Australia) and height was measured to the nearest $0.1 \mathrm{~cm}$ using a wall-mounted stadiometer (Holtain Instruments Ltd, Crymych, Dyfed, UK). BMI was calculated as weight divided by height squared. Height, weight and BMI Z-scores were calculated using the Centers for Disease Control and Prevention LMS values ${ }^{(15)}$. Percentages of ideal body weight for height (IBW) and weight change over the previous month were also calculated ${ }^{(11)}$. Serum albumin concentration $(\mathrm{g} / \mathrm{l})$ was recorded from clinical blood analysis.

\section{Arm anthropometry}

MUAC and triceps skinfold were measured by the same investigator (A. J. M.) at the halfway point between the acromion and olecranon process on the non-dominant side of the body. MUAC was measured with a fibreglass tape to the nearest $0.1 \mathrm{~cm}$ and triceps skinfolds were measured with a Holtain caliper. All measurements were taken twice and averaged by the same observer. MUAC and triceps skinfold were converted to $Z$-scores using the reference data published by Frisancho ${ }^{(16)}$.

\section{Body cell mass}

Potassium is the primary intracellular cation, and as $98 \%$ of the body's potassium is located within the $\mathrm{BCM}^{(17)}$, it is possible to determine the BCM from TBK analysis. TBK analysis was performed using a shadow-shield whole-body counter (AccuScan; Canberra Industries, Boston, MA, USA) that contains three sodium iodide crystal scintillation detectors arranged above a scanning bed. The crystals detect the $1.46 \mathrm{MeV} \gamma$-rays being emitted by the potassium- 40 found in the body. As a fixed proportion of the body's potassium occurs as the natural isotope potassium-40, TBK can be determined.

The measurement of TBK required the subject to lie supine on a bed that is moved under the detectors. Two $1067 \mathrm{~s}$ scans were performed for each subject with all personal metallic objects having been removed. Background and sensitivity checks were completed daily and considered in each measurement, with TBK being reported in grams. BCM was then calculated from TBK using the equation of Wang et al. ${ }^{(18)}$ :

$$
\mathrm{BCM}(\mathrm{kg})=\frac{\operatorname{TBK}(\mathrm{g}) \times 9 \cdot 18}{39 \cdot 1} .
$$

The amount of BCM is related to height, with BCM increasing with growth in height, regardless of improving nutritional status. Therefore, BCM was adjusted for height (BCMI), with height being raised to the power of 2.5 for females and 3 for males $^{(19)}$. The BCMI were then converted to $Z$-scores based on our laboratory's reference of 313 children.

\section{Statistical analysis}

Means and standard deviations were used to describe the population sample. In the present study, we have chosen a cut-off of -1.65 SD to determine those individuals who were malnourished when expressed as the BCMI Z-score. This cut-off is equivalent to the 5 th percentile. Indicators of poor nutritional status in the other measures were $<90 \%$ IBW for height, albu$\min <35 \mathrm{~g} / \mathrm{l},>\% 5$ weight loss in the previous month, and BMI, MUAC and triceps $Z$-score $<-1.65^{(1,10)}$

Pearson's correlation was conducted between BCMI and simple variables to examine whether there was a significant relationship. To further examine whether there was a difference in the simple measurements between the well-nourished and poorly nourished patients, the subjects were divided into poorly nourished and well-nourished groups based on the BCMI $Z$-score $(>-1.65)$. Independent Student's $t$ tests were conducted between the two groups for the simple variables. Significance was set at $P<0 \cdot 05$. The statistical package for social sciences for Windows software (SPSS version 13.0) was used for all analyses.

\section{Results}

Forty children, eighteen females and twenty-two males, between 5.4 and 16.4 years participated in the study. Thirtyseven children had an oncological condition and three children required a bone marrow transplant for other clinical conditions. Five patients did not have a triceps skinfold measurement and one patient did not have a MUAC taken; there were forty data points for each other measurement. The average time since initial diagnosis was 1.3 years and ranged between $0 \cdot 1$ and $10 \cdot 8$ years.

Nutritional characteristics of the population are shown in Table 1 . The study population's height and weight $Z$-scores did not differ significantly from zero. Using the BCMI $Z$-score cut-off of $-1 \cdot 65$, nineteen of the children or $48 \%$ were considered malnourished. Nine patients were considered to have a poor nutritional status according to the percentage of IBW, three patients according to the BMI Z-scores, and none 
Table 1. Characteristics of the study population (forty children) (Mean values and standard deviations)

\begin{tabular}{lcc}
\hline & Mean & SD \\
\hline Age (years) & 10.3 & 3.3 \\
$\mathrm{Ht}(\mathrm{cm})$ & 140.7 & 0.2 \\
$\mathrm{Ht} \mathrm{Z}$-score & 0.08 & 0.91 \\
$\mathrm{Wt}(\mathrm{kg})$ & 40.75 & 18.05 \\
Wt Z-score & 0.48 & 1.17 \\
BMl (kg/m $\left.{ }^{2}\right)$ & 19.6 & 4.2 \\
BMl Z-score & 0.62 & 1.26 \\
\%lBW & 109 & 21 \\
Percentage of Wt change & +1.6 & 3.7 \\
MUAC (cm) & 22.8 & 4.5 \\
MUAC Z-score & 0.71 & 1.21 \\
Triceps skinfold (mm) & 16 & 6 \\
Triceps Z-score & 0.98 & 1.20 \\
BCMl Z-score & -1.22 & 1.81 \\
Albumin (g/l) & 41 & 3 \\
\hline
\end{tabular}

\%IBW, percent ideal body weight for height; MUAC, mid-upper arm circumference; BCMI, body cell mass index.

according to the albumin levels, MUAC Z-score or triceps $Z$-score. Only one patient had lost more than $5 \%$ of their body weight in the previous month.

Correlation showed that there was a significant statistical relationship between the BCMI and BMI $Z$-scores $(r=0 \cdot 36$, $P=0.02)$ and the BCMI $Z$-score and triceps skinfold ( $r=-0 \cdot 37, P=0.03$ ). However, these relationships were not considered biologically significant with up to $87 \%$ of the variation in the BCMI $Z$-score not accounted for. There was no significant relationship between BCMI Z-score and percentage of IBW, percentage of weight change, MUAC, MUAC $Z$-score, triceps $Z$-score or albumin, when analysed in the total study population (Table 2).

When divided into two groups based on the nutritional status indicated by the BCMI Z-score, the independent $t$ tests between the well-nourished ( $>-1.65$ BCMI $Z$-score) and malnourished $(<-1.65$ BCMI $Z$-score) groups demonstrated that there was a significant difference in BMI $Z$-scores $(P=0 \cdot 01)$ between the groups. There was no significant difference between the two groups for any of the other simple variables (Table 3).

\section{Discussion}

There is much research focusing on paediatric cancer; however, an aspect of supportive care that has had little

Table 2. Pearson's correlation between body cell mass index (BCMI) $Z$-score and simple nutritional measures

\begin{tabular}{lcc}
\hline & $R^{*}$ & $P$ \\
\hline BMI Z-score & 0.36 & 0.02 \\
\%IBW & 0.23 & 0.15 \\
Percentage of Wt change & 0.002 & 0.99 \\
MUAC (cm) & -0.14 & 0.40 \\
MUAC Z-score & 0.22 & 0.18 \\
Triceps skinfold (mm) & -0.37 & 0.03 \\
Triceps Z-score & -0.14 & 0.03 \\
Albumin (g/l) & -0.04 & 0.81 \\
\hline
\end{tabular}

\%IBW, percent ideal body weight for height; MUAC, mid-upper arm circumference. ${ }^{\star}$ Correlation coefficient. recognition is the assessment of nutritional status. Despite the identified problem of poor nutritional status in oncology patients, many centres do not undertake nutritional assessment, and if they do, they only utilise simple indices of nutritional status ${ }^{(1,2,8)}$. Although simple measures are recommended in this population, no studies have evaluated the validity of these methods. The aim of the present paper was to determine whether simple nutritional variables could accurately identify poor nutritional status in children being treated for oncological conditions. Despite half of our paediatric oncology population being identified as having a poor nutritional status using the BCMI Z-score, the simple measures failed to identify poor nutritional status accurately in this population.

The use of weight indices are the most commonly applied nutritional measurement. The BMI Z-score has been shown to be the preferred index in 10- to 18-year-old children with cancer when compared with other weight indices ${ }^{(1)}$. In the present study, only $5 \%$ of the subjects were identified as having a poor nutritional status according to a BMI Z-score cut-off of below -1.65 , compared with the $48 \%$ identified by the BCMI Z-score of $-1 \cdot 65$. When further analysis was done, there was a significant statistical relationship between the BCMI and BMI Z-scores; however, $87 \%$ of biological variance is not accounted for in this relationship, which makes it inappropriate for clinical application. When the subjects were divided according to the nutritional status, the mean BMI Z-scores of the groups were significantly different. The present study showed that although the BMI Z-score was the measure that best represented poor nutritional status compared with the other simple measures, it was still not considered a strong enough indicator of the nutritional status to be recommended for clinical use.

Other weight and height measures, which are recommended for use in oncology patients, are percentages of IBW and weight loss over 1 month. Neither of these measurements were accurate in determining poor nutritional status in the present study. According to the recommended cut-off for the percentage of IBW of below $90 \%$ and more than $5 \%$ weight loss in the previous month, $23 \%$ of the groups were malnourished according to the percentage of IBW and $3 \%$ according to the percentage of weight change. This means that the poor nutritional status would be undiagnosed in $25-45 \%$ of the children.

Many studies have supported the use of arm anthropometry, including MUAC and triceps skinfold, as a preferred indicator of body composition in oncology patients ${ }^{(2,12,14,20)}$. These studies have recommended the measurement of arm anthropometry based on a comparison with weight indices and not reference measures of poor nutritional status. Garofolo et al. ${ }^{(12)}$ recorded the weight for height $Z$-scores, BMI percentiles, triceps skinfolds and MUAC in 127 oncology paediatric patients. They found that weight measurements underestimated the poor nutritional status prevalence according to arm anthropometry. Smith et al. ${ }^{(2)}$ also found that weightrelated indices of nutritional status are unreliable in children with cancer, and that arm anthropometry is a better indication. The present study showed that MUAC and triceps skinfold are not suitable for representing the nutritional status, compared with BCMI $Z$-score. There was a significant statistical negative correlation between BCMI Z-scores and triceps skinfolds in our population, but this was not biologically significant. 
Table 3. Comparison of well-nourished and malnourished subjects (independent Student's $t$ test) ${ }^{*}$

(Mean values and standard deviations)

\begin{tabular}{|c|c|c|c|c|c|}
\hline & \multicolumn{2}{|c|}{ Malnourished ( $n$ 19) } & \multicolumn{2}{|c|}{ Well-nourished ( $n$ 21) } & \multirow[b]{2}{*}{$P$} \\
\hline & Mean & SD & Mean & SD & \\
\hline BMI Z-score & 0.07 & 1.50 & $1 \cdot 12$ & 0.72 & 0.01 \\
\hline$\%$ IBW & 105 & 24 & 114 & 18 & 0.19 \\
\hline Percentage of Wt change & +1.5 & 4.1 & +1.6 & 3.4 & 0.98 \\
\hline $\operatorname{MUAC}(\mathrm{cm})$ & $23 \cdot 2 \dagger$ & 4.8 & $18 \cdot 3$ & $4 \cdot 3$ & 0.70 \\
\hline MUAC Z-score & $0.19 \dagger$ & 1.31 & 0.60 & $1 \cdot 12$ & 0.30 \\
\hline Triceps skinfold (mm) & $17.9 \ddagger$ & $6 \cdot 5$ & $15 \cdot 2 \S$ & $5 \cdot 1$ & 0.10 \\
\hline Triceps Z-score & $0.81 \ddagger$ & 1.25 & $0.76 \S$ & 1.23 & 0.88 \\
\hline Albumin & 41 & 3.5 & 41 & $3 \cdot 2$ & 0.88 \\
\hline
\end{tabular}

\%IBW, percent ideal body weight for height; MUAC, mid-upper arm circumference.

${ }^{*}$ Malnourished body cell mass index $Z$-score $<-1.65$.

$\dagger$ Eighteen subjects only.

$\ddagger$ Sixteen subjects only.

$\S$ Nineteen subjects only.

Albumin is the most commonly utilised biochemical index of nutritional assessment despite being affected by hydration status and liver function ${ }^{(21)}$. Kibirige et al. ${ }^{(22)}$ found that a serum albumin of $31 \mathrm{~g} / \mathrm{l}$ was an indicator of severe weight loss in twenty-four children with leukaemia. While Merritt et al. ${ }^{(13)}$ found that albumin was not associated with weight, height, arm muscle area and triceps skinfold measurements, and did not reflect a depletion in body mass in ninety paediatric oncology patients. The present study found that serum albumin was not a valid indicator of poor nutritional status in a paediatric oncology population and should not be used in this population.

The newly developed paediatric- and sex-specific BCMI $Z$-scores were used in the present study as the reference indicator of poor nutritional status ${ }^{(19)}$. Although it is not the only measure, $\mathrm{BCM}$ was considered to be the ideal reference method for poor nutritional status because it indicates a loss of functional cell mass and is not affected by hydration status. BCM has been shown to be more sensitive than BMI in monitoring changes in muscle mass and protein tissues ${ }^{(23)}$. As malnutrition in children with cancer is usually caused by reduced physical activity, exposure to corticosteroids or reduced intake (all of which will be reflected in altered lean tissue mass), BCMI is a useful measure in these patients. Simple measures such as BMI will only indicate weight or body size changes, not body composition changes.

As with all methods, TBK conversion to BCM has a limitation. There is currently no paediatric-specific equation available to convert TBK into BCM. The equation of Wang et al. ${ }^{(18)}$ used to convert TBK into BCM was formulated using the data of healthy adults, and thus is based on assumptions that may not apply to children with a clinical condition. Therefore, this conversion may be a source of error in the present study.

The simple measures may not have performed well in the present study because they are indicators of body size, not body composition. Poor nutritional status is a loss of functional tissues, which is impossible to determine from general anthropometric measurements. Weight and size measurements cannot distinguish between fat and functional tissue, so weight and size changes may be indicative of hydration changes, fat compartment changes or tumour mass, rather than changes to the function mass. For example, many of our patients who had high BMI were the most poorly nourished ones according to BCMI, and a simple anthropometric measurement would have labelled them as overweight and their loss of functional tissue would have gone unidentified.

A limitation of the present study lies within the reference populations. The reference population used to formulate the BCMI Z-scores is 313 children, which represents only a small sample at any given age. On the other hand, the reference data for the anthropometry Z-scores are significantly larger and ethnically different. This difference may contribute in part to the lack of relationship seen between the methods in the present study.

Despite simple measures being widely recommended to evaluate the nutritional status in paediatric oncology patients, the present study shows that simple nutritional measurements will not accurately identify patients who have a poor nutritional status. The prognosis and recovery of children being treated for oncological conditions will be severely affected if poor nutritional status goes undetected. The implication of the present study's findings is that the simple measurement should not be used only to assess the nutritional status in oncology patients but that more detailed alternatives need to be evaluated for clinical use in this population.

\section{Acknowledgements}

The authors wish to thank the Royal Children's Hospital Foundation for their financial support of the Children's Nutrition Research Center. The authors have no personal, commercial, political, academic or financial conflicts of interest in this research. A. J. M. was responsible for the subject testing, research design, interpretation of the results and writing of the manuscript. M. W. was responsible for subject recruitment, research design and review of the manuscript. P. S. W. D. was responsible for the statistics and contributed to the interpretation of results and review of the manuscript.

\section{References}

1. Pietsch JB \& Ford C (2000) Children with cancer: measurements of nutritional status at diagnosis. Nutr Clin Pract $\mathbf{1 5}$, $185-188$. 
2. Smith DE, Stevens MC \& Booth IW (1991) Malnutrition at diagnosis of malignancy in childhood: common but mostly missed. Eur J Pediatr 150, 318-322.

3. Lobato Mendizábal E, Ruiz-Argüelles GJ \& Marín-López A (1989) Leukaemia and nutrition I: malnutrition is an adverse prognostic factor in the outcome of treatment of patients with standard-risk acute lymphoblastic leukaemia. Leuk Res 13, 899-906.

4. Mejia-Arangure JM, Fajardo-Gutierrez A, Reyes-Ruiz NI, Bernaldez-Rios R, Mejia-Dominguez AM, Navarrete-Navarro S \& Martínez-García MC (1999) Malnutrition in childhood lymphoblastic leukemia: a predictor of early mortality during the induction-to-remission phase of the treatment. Arch Med Res 30, $150-153$.

5. Reilly JJ, Odame I, McColl JH, McAllister PJ, Gibson BE \& Wharton BA (1994) Does weight for height have prognostic significance in children with acute lymphoblastic leukemia? Am J Pediatr Hematol Oncol 16, 225-230.

6. Viana MB, Murao M, Ramos G, Oliveira HM, de Carvalho RI, de Bastos M, Colosimo EA \& Silvestrini WS (1994) Malnutrition as a prognostic factor in lymphoblastic leukaemia: a multivariate analysis. Arch Dis Child 71, 304-310.

7. Khan AU, Sheikh MU \& Intekhab K (2006) Pre-existing malnutrition and treatment outcome in children with acute lymphoblastic leukaemia. J Pak Med Assoc 56, 171-173.

8. Ladas EJ, Sacks N, Brophy P \& Rogers PC (2006) Standards of nutritional care in pediatric oncology. Pediatr Blood Cancer 46, 339-344.

9. Murphy AJ, Wells JC, Williams JE, Fewtrell MS, Davies PS \& Webb DK (2006) Body composition in children in remission from acute lymphoblastic leukemia. Am J Clin Nutr 83, 70-74.

10. Sala A, Antillon F, Pencharz PB \& Barr R (2005) Nutritional status in children with cancer: a report from the AHOPCA workshop. Pediatr Blood Cancer 45, 230-236.

11. McCallum P \& Polisena C (editors) (2000) The Clinical Guide to Oncology Nutrition. Chicago, IL: Oncology Nutrition Dietetic Group, The American Dietetic Association.
12. Garofolo A, Lopez FA \& Petrilli AS (2005) High prevalence of malnutrition among patients with solid non-hematological tumors as found by using skinfold and circumference measurements. Sao Paulo Med J 123, 277-281.

13. Merritt RJ, Kalsch M, Roux LD, Ashley-Mills J \& Siegel SS (1985) Significance of hypoalbuminemia in pediatric oncology patients - malnutrition or infection? J Parenter Enteral Nutr 9, 303-306.

14. Oguz A, Karadeniz C, Pelit M \& Hasanoglu A (1999) Arm anthropometry in evaluation of malnutrition in children with cancer. Pediatr Hematol Oncol 16, 35-41.

15. National Center for Health Statistics (2000) CDC Growth Charts. Selected Percentiles and LMS Parameters. Hyattsville, MD: National Center for Health Statistics.

16. Frisancho AR (1990) Anthropometric Standards for the Assessment of Growth and Nutritional Status. Ann Arbor, MI: University of Michigan Press.

17. Moore FD (1980) Energy and the maintenance of the body cell mass. JPEN J Parenter Enteral Nutr 4, 228-260.

18. Wang Z, Saintonge MP, Lecumberri B, et al. (2004) Body cell mass: model development and validation at the cellular level of body composition. Am J Physiol Endocrinol Metab 286, E123-E128

19. Murphy AJ \& Davies PS (2008) Body cell mass index in children: interpretation of total body potassium results. $\mathrm{Br} J \mathrm{Nutr}$ 100, 666-668.

20. Brennan BM \& Thomas AG (1997) Nutritional status in children with acute leukemia. J Pediatr Gastroenterol Nutr 25, 248-249.

21. Altman A (editor) (2004) Supportive Care of Children with Cancer. Baltimore, MD: The Johns Hopkins University Press.

22. Kibirige MS, Morris Jones PH \& Stevens RF (1987) Indicators of malnutrition in leukaemic children. Arch Dis Child 62, 845-846.

23. Talluri A, Liedtke R, Mohamed EI, Maiolo C, Martinoli R \& De Lorenzo A (2003) The application of body cell mass index for studying muscle mass changes in health and disease. Acta Diabetol 40, Suppl. 1, S286-S289. 